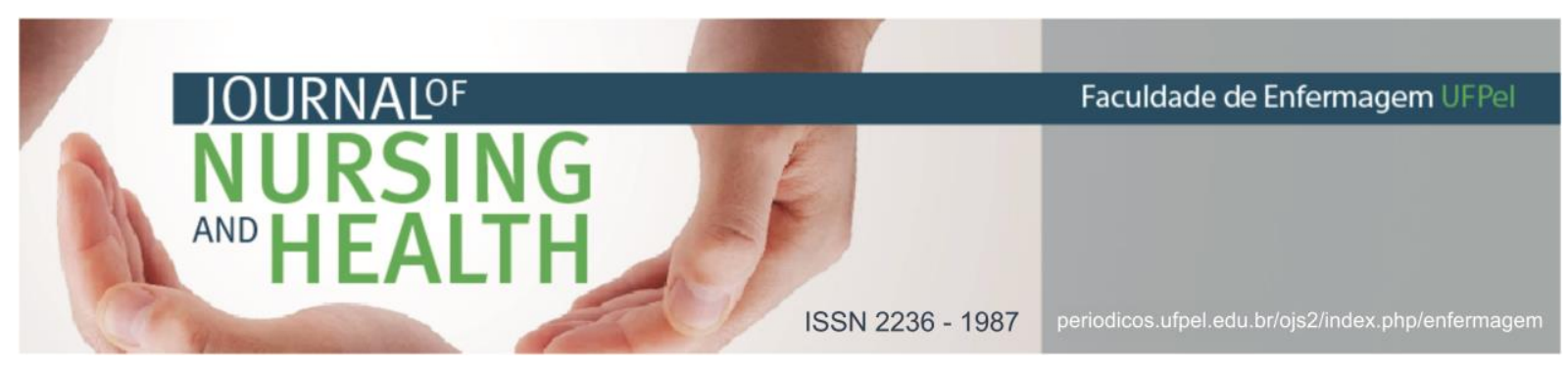

RELATO DE EXPERIÊNCIA

\title{
Atividade educativa para identificação correta do paciente: um relato de experiência
}

\author{
Educational activity for correct patient identification: an experience report \\ Actividad educativa para la identificación correcta del paciente: un relato de
experiencia
}

Campelo, Rômulo Costa$^{1}$; Silva, Walicy Cosse²; Sousa, Carla Kellen Lima3; Araújo, Gilsara Leite ${ }^{4}$; Bizerra, Laureany5; Leite, André Geisteira de Moura6; Araújo, Raquel Vilanova7

Como citar este artigo: Campelo RC, Silva WC, Sousa CKL, Araújo GL, Bizerra L, Leite AGM et al. Atividade educativa para identificação correta do paciente: um relato de experiência. J nurs health. 2018;8(3):e188305

\section{RESUMO}

Objetivo: descrever a experiência de acadêmicos de Enfermagem no desempenho da Atividade Educativa sobre a identificação correta do paciente. Metódos: trata-se de um relato de experiência no qual é descrito a atividade desenvolvida acerca da identificação correta do paciente. A ação foi direcionada aos acompanhantes dos pacientes e foi desenvolvida em quatro etapas distintas: escolha da temática; planejamento; execução e observação da realidade e; levantamento e discussão de problemas. Resultados: foi possível perceber o envolvimento e participação dos acompanhantes e pacientes na dinâmica proposta pelo grupo, sendo visto maior entendimento sobre a temática discutida. Conclusão: portanto, verificou-se a importância da identificação correta do paciente e a dupla checagem para manutenção de sua segurança e qualidade da assistência.

Descritores: Segurança do paciente; Sistemas de identificação de pacientes; Cuidados de enfermagem.

\section{ABSTRACT}

Objective: the purpose of this study was to describe the experience of Nursing students in the performance of the Educational Activity on the correct identification of the patient. Methods: it is an experience report in which the activity developed about the correct identification of the patient is described. The action was directed to the patients' companions and was developed in four distinct

\footnotetext{
1 Discente do curso de Bacharelado em Enfermagem. Centro Universitário Santo Agostinho (UNIFSA). E-mail: romulocam14@gmail.com http://orcid.org/0000-0002-881

2 Discente do curso de Bacharelado em Enfermagem. Centro Universitário Santo Agostinho (UNIFSA). E-mail: cosse.wallyce174.wc@gmail.com http://orcid.org/0000-0001-9338-9067

3 Discente do curso de Bacharelado em Enfermagem. Centro Universitário Santo Agostinho (UNIFSA). E-mail: carlakellenenfermagem@gmail.com http: //orcid.org/0000-0002-8000-2414

4 Discente do curso de Bacharelado em Enfermagem. Centro Universitário Santo Agostinho (UNIFSA). E-mail: gsaraleite@gmail.com http://orcid.org/0000-0003-3319-1088

5 Discente do curso de Bacharelado em Enfermagem. Centro Universitário Santo Agostinho (UNIFSA). E-mail: laureanybezerra@outlook.com http://orcid.org/0000-0003-0645-6239

6 Discente do curso de Bacharelado em Enfermagem. Centro Universitário Santo Agostinho (UNIFSA). E-mail: gilsaraleite@hotmail.com http://orcid.org/0000-0002-9254-5248

7 Enfermeira. Mestre em Ciências e Saúde. Centro Universitário Santo Agostinho (UNIFSA). E-mail: raquelvila@outlook.com http://orcid.org/0000-0001-5902-9869
} 


\section{JOURNALOF \\ IsS $2236-1987$}

stages: choice of thematic; planning; execution and observation of reality and survey and discussion of problems. Results: it was possible to perceive the involvement and participation of the companions and patients in the dynamics proposed by the group, with a greater understanding of the topic discussed. Conclusion: therefore, it was verified the importance of the correct identification of the patient and double checking to maintain their safety and quality of care.

Descriptors: Patient safety; Patient identification systems; Nursing care.

\section{RESUMEN}

Objetivo: el estudio tuvo como objetivo describir la experiencia de académicos de Enfermería en el desempeño de la Actividad Educativa sobre la identificación correcta del paciente. Métodos: se trata de un relato de experiencia en el que se describe la actividad desarrollada acerca de la identificación correcta del paciente. La acción fue dirigida a los acompañantes de los pacientes y fue desarrollada en cuatro etapas distintas: elección de la temática; planificación; ejecución y observación de la realidad y levantamiento y discusión de problemas. Resultados: fue posible percibir la participación de los acompañantes y pacientes en la dinámica propuesta por el grupo, siendo visto mayor entendimiento sobre la temática discutida. Conclusión: por lo tanto, se verificó la importancia de la identificación correcta del paciente y el doble chequeo para mantener su seguridad y calidad de la asistencia.

Descriptores: Seguridad del paciente; Sistemas de identificación de pacientes; Atención de enfermería.

\section{INTRODUÇÃO}

Desde 2000, a temática sobre segurança do paciente tornou-se foco de grande interesse pelos pesquisadores em todo o mundo. Assim, o tema se tornou uma indagação da sociedade e vem sendo compreendida ao longo do tempo como de extrema importância para a diminuição do risco de danos desnecessários oferecidos pelos serviços de saúde. Em 2013, no Brasil, foi lançado o Programa Nacional de Segurança do Paciente, que veio com a proposta de metas a serem implantadas nos serviços de saúde, buscando a melhoria da segurança. Sendo evidente a necessidade da colaboração de toda a equipe de modo a prevenir ao máximo as ações inseguras prestadas pela a equipe de saúde. ${ }^{1-2}$

A segurança do paciente é de suma importância para a redução dos riscos de danos e lesões, obedecendo aos princípios proposto pela Organização Mundial da Saúde (OMS) e pela Classificação Internacional de Segurança do Paciente (ICPS). ${ }^{3}$

O Núcleo de Segurança do Paciente (NSP) foi também criado com a finalidade de proporcionar ações seguras e prestação de serviços assistenciais qualificados, vem sendo compreendido e trabalhado com intuito de promover uma adaptação consistente ao plano de segurança ao paciente. Assim, são trabalhadas metas que tem como escopo o cumprimento com responsabilidade e dentro dessas encontra-se a identificação do paciente, uma das metas mais implantadas com sucesso nos hospitais. ${ }^{4}$

Com a elevada disseminação acerca da cultura de segurança do paciente 0 entendimento sobre 0 assunto tem evoluído, permitindo maior compreensão e percepção por 
ISSN 2236 - 1987

parte dos profissionais de saúde, passa a ser uma qualidade fundamental no cuidado. Visto que o evento adverso reflete economicamente nos custos sociais, pode ainda desencadear no cliente sequelas irreversíveis e que repercute também em suas famílias, constituindo consequentemente um problema de saúde pública. ${ }^{5}$

No entanto, apesar de avanços, o hospital apresenta-se como um local inseguro e perigoso. As condições algumas vezes precárias também se relacionam a superlotação de hospitais, poucos recursos financeiros para melhorias, insuficiência no número de leitos, dupla jornada de trabalho, a falha no dimensionamento do pessoal entre outros, contribuem para o aumento dos riscos de eventos adversos, comprometendo a segurança e vida do paciente. Diante desses fatores pertinentes à profissão, é notória a importância que o enfermeiro realize capacitação no assunto, pois ao melhorar seu conhecimento sobre a aplicabilidade de indicadores que avaliam o processo e os efeitos que 0 erro pode repercutir, possibilitará um olhar mais aguçado e amplo para a qualidade da assistência e segurança do paciente. ${ }^{6}$

Destacam-se nesse cenário os chefes e gerentes de saúde, que têm o papel legal e moral de assegurar uma assistência responsável e de alta qualidade, visando sempre se aperfeiçoar no cuidado oferecido ao cliente. Visto que as pessoas que estão na gestão do cuidado têm a responsabilidade de disseminar a política, procedimentos e sistemas organizacionais que devem ser seguidos no serviço. Alguns estudos demonstraram que os profissionais de saúde concordam que é interessante criar uma cultura aberta e transparente no que se refere a melhorias na segurança do paciente. ${ }^{7}$

Desta maneira, fica claro que a correta abordagem por parte do profissional é decisiva e o princípio da segurança, considerando que à atuação ciente e responsável do profissional são essenciais para minimizar os erros e falhas que ocorrem durante 0 processo assistencial, bem como a comunicação efetiva entre a equipe é a chave para a diminuição dos erros. Mesmo com a enfermagem sendo considerada, após estudos, a profissão que gera mais estresse nos profissionais, devido a assistência prestada 24 horas intermitentemente, resultam em estresse ocupacional, insatisfação profissional, todos eles influenciando negativamente na qualidade da assistência prestada ao cliente por parte da equipe de enfermagem. ${ }^{2,4}$

Assim, é perceptível a importância peculiar da enfermagem na prevenção de eventos adversos, pois além de representar a maior equipe, também pelo fato de estar mais próximo do paciente, tendo mais tempo e consequentemente responsabilidade na prestação de cuidados às necessidades e alterações do paciente, embora não seja responsabilidade exclusiva do enfermeiro, mas de toda a equipe multiprofissional, prestar o cuidado de qualidade ao paciente, assegurando maior segurança ao mesmo. ${ }^{8}$

Assim, cabe ao enfermeiro aprimorar seus conhecimentos na área de segurança do paciente, por meio da 


\section{JOURNALOF \\ NURSING \\ AND HEALTH}

ISSN 2236 - 198

capacitação profissional que deve estar constantemente inserida em sua prática cotidiana. Dessa forma a enfermagem deve estar em constante processo de educação e atualização de forma que possibilite a realização uma assistência mais qualificada, fornecendo apoio para que a segurança e a qualidade da assistência aconteçam de forma eficaz nas instituições de saúde. ${ }^{9}$

Neste cenário, 0 trabalho apresentou como objeto de estudo a identificação dos pacientes, trabalhada no âmbito hospitalar como uma das metas internacionais de segurança do paciente. Apresenta como justificativa a necessidade de obter conhecimento sobre a situação desta meta em específico, dentro de um hospital público de urgência, e ampliar a discussão acerca de sua importância para a garantia de maior segurança ao paciente no período de internação. 0 estudo tem por objetivo descrever a experiência dos acadêmicos de enfermagem no desenvolvimento de atividade educativa relacionada a identificação correta do paciente em um hospital de referência.

\section{MATERIAIS E MÉTODOS}

Trata-se de um relato de experiência de discentes do Curso de Enfermagem relacionada à Atividade Educativa desenvolvida em um Hospital de Urgência da Capital do Piauí, na oportunidade do estágio curricular I, sob supervisão da preceptora, sendo realizado em março de 2018. Caracteriza-se ainda como um hospital de grande porte, que oferece suporte às atividades de ensino, pesquisa e extensão às Instituições de ensino da rede pública e privada do estado.

A temática abordada foi sobre a identificação do paciente, a meta 1 do protocolo de segurança do paciente, e teve como norteador teórico o Protocolo Operacional Padrão (POP) de identificação do paciente da própria instituição. A finalidade da Atividade Educativa foi conscientizar os acompanhantes dos pacientes sobre a importância da identificação correta do paciente, o uso da pulseira de identificação e manutenção da qualidade da assistência prestada ao indivíduo durante 0 processo de hospitalização.

A atividade foi desenvolvida em quatro etapas distintas, sendo a Etapa 1 - Escolha da temática, Etapa 2 Planejamento, Etapa 3 - Execução e observação da realidade e Etapa 4 Levantamento e discussão de problemas.

Nas etapas 1 e 2 foram realizadas reuniões com o grupo de discentes e supervisora e encontros com os profissionais do Núcleo de Educação Continuada da Instituição de Saúde que apoiam e ofereceram suporte para o desenvolvimento da Atividade Educativa. No hospital, as seis metas internacionais de segurança do paciente já foram implantadas a partir do Núcleo de Segurança do Paciente (NSP), setor responsável pela supervisão e controle da aplicação das metas nos diversos departamentos da instituição. Foram realizadas discussões sobre o tema, levantamento bibliográfico, construção de material informativo e ilustrativo, cartazes, escolha de logomarca para camisetas e 
planejamento da dinâmica a ser realizada com os acompanhantes.

$\mathrm{Na}$ etapa 3, parte do grupo teve como competência a execução da Atividade Educativa nas enfermarias, de modo a orientar os acompanhantes sobre a importância do uso da pulseira de identificação, informar sobre a rotina para a troca da pulseira, a importância da identificação correta do paciente e do leito de internação. As orientações foram realizadas com auxílio de cartazes, placas, pulseira de identificação e entrega de material informativo.

Durante a Atividade Educativa foram abordados aspectos sobre como é a pulseira de identificação; sua finalidade; quais dados devem constar na pulseira de identificação; a importância da dupla checagem na realização de procedimentos, exames e administração de medicamentos e quando, como e para quem solicitar a substituição da pulseira de identificação.

Ao final da Atividade Educativa, foi realizado uma dinâmica com os acompanhantes a fim de esclarecer possíveis dúvidas acerca do tema abordado. Os acompanhantes foram convidados a retirar da caixa um papel e responder 'a pergunta relacionada ao tema abordado, na oportunidade, foi reforçado mais alguns aspectos importantes e entregue um brinde.

A outra parte do grupo fez um levantamento acerca da realidade das clínicas em relação à identificação de pacientes, troca das pulseiras danificadas, identificação dos pacientes e dos leitos correspondentes. Por fim na Etapa 4, realizou-se nova reunião com o grupo e preceptora para levantamento dos problemas encontrados para apresentação e discussão dos mesmos em quadros e tabelas juntamente com a equipe de Educação Continuada.

\section{RESULTADOS}

A atividade Educativa foi realizada nas enfermarias dos postos 01,02 e 03 . O posto 1 é caracterizado pela internação de pacientes com cardiopatias, insuficiência renal e problemas oftalmológicos. 0 posto 2 encontra-se pacientes com estado de saúde mais crítico e instáveis, a maioria vítimas de traumas. Já no posto 3 , são realizadas internações de pacientes com problemas vasculares.

A Atividade Educativa foi realizada com os acompanhantes dos 44 pacientes que se encontravam internados de acordo com a Tabela 1.

Tabela 1: Número de pacientes abordados na ação por posto de enfermagem. Hospital de Urgência, Teresina-PI, 2018.

\begin{tabular}{cc}
$\begin{array}{c}\text { Posto de } \\
\text { Enfermagem }\end{array}$ & $\begin{array}{c}\text { Número de } \\
\text { Pacientes }\end{array}$ \\
\hline 1 & 13 \\
2 & 13 \\
3 & 18 \\
\hline Total & 44
\end{tabular}

Fonte: Pesquisa direta, 2018.

No desenvolvimento da Atividade Educativa percebeu-se a boa receptividade, interesse e envolvimento dos acompanhantes e inclusive de alguns pacientes com os alunos e com a preceptora. Durante as discussões foram demonstradas preocupações relacionadas ao tema. Ao final foi possível perceber o 


\section{JOURNALOF \\ NURSING \\ AND}

envolvimento e participação dos acompanhantes e pacientes na dinâmica proposta pelo grupo, sendo visto maior entendimento sobre a temática discutida.

De acordo com o Figura 1, observa-se que mais da metade dos pacientes, isto é, 25 pessoas que representa (57\%) encontravam-se sem a pulseira de identificação.

Figura 1: Percentual de pacientes que estavam com e sem uso da pulseira de identificação durante a ação. Hospital de Urgência, Teresina-PI, 2018.

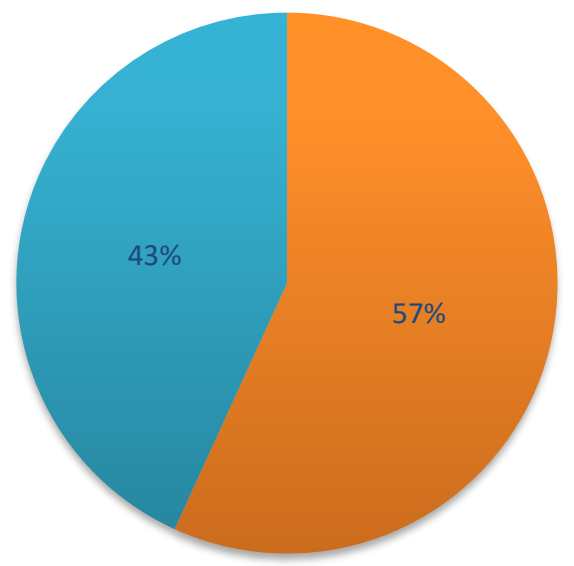

— Pacientes sem pulseira de identificação

— Pacientes com pulseira de identificação

Fonte: Pesquisa direta, 2018.

\section{DISCUSSÃO}

A Política Nacional de Segurança do Paciente (PNSP) apresenta seis importantes protocolos para assegurar a segurança do paciente durante sua internação hospitalar. São eles: identificar corretamente o paciente; melhorar a comunicação entre profissionais de Saúde; melhorar a segurança na prescrição, no uso e na administração de medicamentos; assegurar cirurgia em local de
ISSN 2236 - 198

intervenção, procedimento e paciente corretos; higienizar as mãos para evitar infecções e reduzir o risco de quedas e lesão por pressão. ${ }^{10}$

Atualmente, as falhas no processo de identificação do paciente tem sido uma das preocupações no ambiente hospitalar, e importante fator de risco para aumento de eventos adversos. Destaca-se então que o cumprimento deste protocolo é fundamental e de responsabilidade de todos os profissionais. $\mathrm{Na}$ oportunidade da Atividade Educativa foi possível observar que 25 (57\%) pacientes, encontravam-se nos postos de internação sem a pulseira de identificação. Esse resultado é preocupante, pois o Serviço de Educação Continuada realiza ações frequentes de conscientização dos profissionais quanto a importância do seu uso, além de fazer parte da rotina assistencial. 0 resultado evidencia falha no processo de execução da meta 1 do PNSP, tornando 0 paciente vulnerável a riscos que podem agredir a sua e prolongar o tempo de internação. ${ }^{10}$

As políticas e procedimentos são instrumentos utilizados para auxiliar na proteção dos pacientes, evitando causar danos durante a prestação do cuidado. Dessa forma, é fundamental que essas políticas sejam implementadas e seguidas, com intuito de promover a segurança do paciente e prevenir danos evitáveis. Neste contexto, é indispensável que os profissionais de saúde tenham o aprendizado e a compreensão acerca da importância da identificação correta do paciente para evitar a ocorrência de erros e complicações 


\section{ISSN 2236 - 1987}

que ofereçam riscos à segurança dos indivíduos internados. ${ }^{11}$

Sendo assim, o uso da pulseira de identificação é essencial para a garantia da segurança dos pacientes durante a prestação de cuidados nas dependências hospitalares. Assim, ressalta-se a importância das atividades educativas direcionadas aos acompanhantes/cuidadores, como a que foi realizada, de modo a proporcionar conhecimento aos mesmos e bem como, aos profissionais a fim de aumentar a responsabilidade de ambos e reduzir os acidentes e danos relacionados a assistência à saúde. ${ }^{12}$

Os serviços de saúde têm utilizados diversos métodos na identificação do paciente, incluindo placas de cabeceira, crachás e pulseiras. No local onde a atividade foi realizada, a identificação é realizada por meio de pulseiras e placas de cabeceira, no entanto, foram observadas falhas na identificação dos pacientes, como a ausência da pulseira e na legibilidade da pulseira. ${ }^{13}$

Neste âmbito, o engajamento e compromisso dos profissionais de enfermagem é importante para reduzir os riscos e os danos relacionados aos cuidados a saúde, de modo a manter a segurança do paciente e melhorar a qualidade da assistência. Para isto, destaca-se a importância do desenvolvimento das atividades de educação em saúde direcionadas não apenas aos profissionais da enfermagem, mas a todos envolvidos com a assistência e o cuidado, de modo a reduzir os eventos adversos no meio hospitalar. ${ }^{14}$

\section{CONCLUSÃO}

Durante a Atividade Educativa, foi possível identificar algumas inconformidades e superficionalidades na aplicação dos protocolos assistenciais, assim como da falta de motivação e de colaboração dos profissionais para a efetiva aplicação do mesmo na rotina assistencial. Observou-se que muitos dos acompanhantes não tinham conhecimento acerca da importância do uso da pulseira de identificação, e demonstraram sentimento de preocupação com as falhas na identificação.

A participação dos discentes possibilitou a oportunidade de uma vivência ímpar e primordial para o processo de trabalho enquanto futuros enfermeiros. Percebeu-se a importância do enfermeiro enquanto gestor da equipe, de modo a motivar e incentivar a participação de todos da equipe assistencial nas atividades educativas ressaltando o compromisso de cada com a integridade do indivíduo/família/comunidade.

0 desenvolvimento da atividade contribuiu para ajudar os discentes a desenvolverem habilidades que só é possível adquirir com a vivência, a experiência, bem como, a autonomia, segurança, criatividade e confiança.

\section{REFERÊNCIAS}

1 Albuquerque AA. Segurança do paciente à luz do referencial dos direitos humanos. Rev direito sanit [Internet]. 2017 [acesso em 2018 out 04];17(2):117-37. Disponível em: http://www.revistas.usp.br/rdisan/ar ticle/view/122309/119046 


\section{ISSN 2236 - 1987}

2 Lemos CS, Cunha KCS. The use of patient identification in a hospital unit. Rev enferm UFPE on line [Internet]. 2017 [cited 2018 Sept 28];11(1):130-9. Available from: https://periodicos.ufpe.br/revistas/r evistaenfermagem/article/view/1188 $6 / 14348$

3 Siman AG, Cunha SGS, Brito MJM. Nursing actions for patient safety in hospitals: integrative review. Rev enferm UFPE on line [Internet]. 2017 [cited 2018 Sept 28];11(2):1016-24. Available from: https://periodicos.ufpe.br/revistas/r evistaenfermagem/article/view/1347 2/16175

4 Azevedo KCC, Alves AMPM, Félix ZC, Viana ACG. Implementation of the patient safety core in a health service. Rev enferm UFPE on line [Internet]. 2016 [cited 2018 Sept 28];10(12):46925. Available from: https://periodicos.ufpe.br/revistas/r evistaenfermagem/article/viewFile/1 $1540 / 13447$

5 Bampi R, Lorenzini E, Krauzer IM, Ferraz L, Silva EF, Agnol CMD. Perspectives of the nursing team on patient safety in an emergency unit. Rev enferm UFPE on line [Internet]. 2017 [cited 2018 Sept 28];11(2):58490. Available from: https://periodicos.ufpe.br/revistas/r evistaenfermagem/article/view/1197 7/14529

6 Araujo MAN, Filho WDL, Silveira RS, Souza JC, Barlem ELD, Teixeira NS. Segurança do paciente na visão de enfermeiros: uma questão multiprofissional. Enferm foco (Brasília) [Internet]. 2017 [acesso em 2018 set 28];8(1):52-6. Disponível em: http://revista.cofen.gov.br/index.php /enfermagem/article/view/984/362

7 Parand A, Dopson S, Renz A, Vincen $C$. The role of hospital managers in quality and patient safety: a systematic review. BMJ open [Internet]. 2014 [cited 2018 Sept 28];4(9):1-15. Available from: https://bmjopen.bmj.com/content/b mjopen/4/9/e005055.full.pdf

8 Trettene AS, Andrade CS, Kostrisch LMV, Tabaquim MLM, Razera APR. Stress in nursing professionals working at a specialized hospital. Rev enferm UFPE on line [Internet]. 2016 [cited 2018 Sept 28];10(12):4450-8. Available from:

https: / periodicos.ufpe.br/revistas/r evistaenfermagem/article/view/1150 9/13386

9 Domingues AL, Santos SVM, Góes FSN, Martinez MR. Avaliação da contribuição da acreditação hospitalar no processo de educação permanente em saúde. Rev enferm UFPE on line [Internet]. 2017 [acesso em 2018 set 28];11(Suppl 5):2177-84 . Disponível em: https://periodicos.ufpe.br/revistas/r evistaenfermagem/article/view/2337 3/19009

10 Nunes CF, Guerra TRB, Dehoul MS, Maior MCGS. Segurança do paciente em uma grande emergência do SUS: como assegurar a prática? Academus revista científica da saúde [Internet]. 2016 [acesso em 2018 out 04];1(1):1-5. Disponível em: https://smsrio.org/revista/index.php /reva/article/view/144/143

11 Lemos GC, Azevedo C, Bernardes MFVG, Ribeiro HCTC, Menezes AC, Mata LRF. The patiente safety culture in the scope of nursing: theoretical 


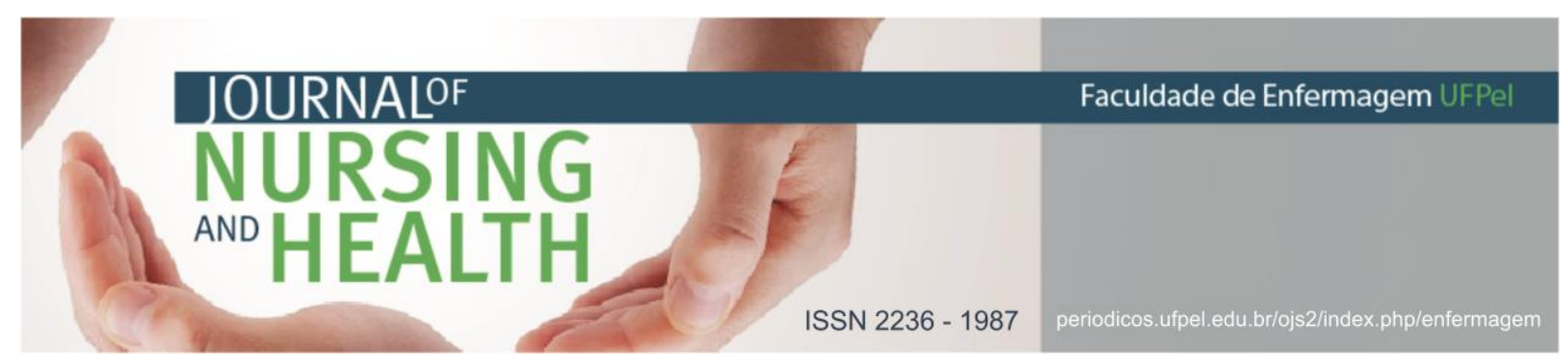

reflection. Rev enferm cent-oeste $\min$ [Internet]. 2018 [cited 2018 Sept 28];8:8-2600. Available from: http: / /www.seer.ufsj.edu.br/index.p $\mathrm{hp} / \mathrm{recom} /$ article/view/2600/1881

12 Macedo MCS, Almeida LF, Assad LG, Rocha RG, Ribeiro GSR, Pereira LMV. Identificação do paciente por pulseira eletrónica numa unidade de terapia intensiva geral adulta. Referência [Internet]. 2017 [acesso em 2018 out 04];4(13): 63-70. Disponível em: http://www.scielo.mec.pt/pdf/ref/vs erlVn13/serIVn13a07.pdf

13 Hoffmeister LV, Moura GMSS. Use of identification wristbands among patients receiving inpatient treatment in a teaching hospital. Rev latino-am enferm [Internet] 2015 [cited 2018 Oct 04];23(1):36-43. Available from: http: //www.scielo.br/pdf/rlae/v23n1 /0104-1169-rlae-23-01-00036.pdf

14 Bezerra ALQ. A segurança do paciente e a enfermagem. Nursing (São Paulo) [Internet]. 2018 [acesso em 2018 out 04];21(239):2091. Disponível em:

http: / /www.revistanursing.com.br/re vistas/239-Abril2018/editorial.pdf

Data de submissão: 05/09/2018

Data de aceite: 26/10/2018

Data de publicação: 31/10/2018 